\title{
Computed Tomography - A powerful imaging technique in the fields of dimensional metrology and quality control
}

\author{
Gabriel Probst*a, Bart Boeckmans ${ }^{\mathrm{b}}$, Wim Dewulf ${ }^{\mathrm{a}}$, Jean-Pierre Kruth ${ }^{\mathrm{b}}$ \\ ${ }^{a}$ KU Leuven, Dept. of Mechanical Engineering, A. 13, B-3000 Leuven, Belgium, \\ ${ }^{b}$ KU Leuven, Dept. of Mechanical Engineering, Celestijnenlaan 300b, B-3001 Leuven, Belgium
}

\begin{abstract}
X-ray computed tomography (CT) is slowly conquering its space in the manufacturing industry for dimensional metrology and quality control purposes. The main advantage is its non-invasive and non-destructive character. Currently, $\mathrm{CT}$ is the only measurement technique that allows full 3D visualization of both inner and outer features of an object through a contactless probing system. Using hundreds of radiographs, acquired while rotating the object, a 3D representation is generated and dimensions can be verified. In this research, this non-contact technique was used for the inspection of assembled components. A dental cast model with 8 implants, connected by a screwed retained bar made of titanium. The retained bar includes a mating interface connection that should ensure a perfect fitting without residual stresses when the connection is fixed with screws. CT was used to inspect the mating interfaces between these two components. Gaps at the connections can lead to bacterial growth and potential inconvenience for the patient who would have to face a new surgery to replace his/hers prosthesis. With the aid of CT, flaws in the design or manufacturing process that could lead to gaps at the connections could be assessed.
\end{abstract}

Keywords: Computed tomography (CT), dimensional metrology, quality control, contactless probing system

\section{INTRODUCTION}

X-ray computed tomography (XCT) applied to coordinate metrology was introduced in industry in the early ' 90 s, however still with a relatively low accuracy $(0,1 \mathrm{~mm})[1,2]$. Only at the beginning of this century, the first dedicated dimensional XCT machine were put on the market. Since then, many other and more powerful industrial XCT systems were introduced by a variety of manufacturers [3].

A big advantage of XCT systems is that they allow performing dimensional quality control and material quality control simultaneously, rather than one or the other as is the case with the more traditional techniques. Another important aspect of XCT is that it also allows the measurement of both inner and outer structures without the need of performing a test of destructive nature. In this sense, XCT is of high interest in the fields of additive manufacturing and $2 \mathrm{~K}$ injection molded plastic parts or plastics with metallic inserts, as it is the only technology that can inspect non-accessible internal features. In these sectors XCT is typically used for the detection of flaws such as voids, inclusions and cracks, and for material analysis. Moreover, XCT is also used for the control of complex assembled components as measuring the individual components of an assembly separately does not guarantee a proper functioning of the assembled system. [3,4]

Besides the aforementioned, XCT is also used in several other sectors for a variety of analyses. Some examples are: the casting and forming industry, the machining (subtractive manufacturing) industry, and the already mentioned additive manufacturing, injection molding and assembly industries. Moreover, XCT is also used for the inspection of electrical and electronic devices, inhomogeneous materials and in the food industry [3,4]. Figure 1 illustrates a few examples where CT is applicable.

In this paper, XCT is used for the inspection of medical implants. Medical implants are devices placed either inside or on the surface of the body to replace, assist or enhance the functionality of some biological structure(s). To address some of the demanding issues related to design, fabrication and implantation the domain of biomanufacturing emerged in 2005 $[6]$.

\footnotetext{
*gabriel.probst@kuleuven.be; phone +32 1637 39 29; www.mech.kuleuven.be
} 


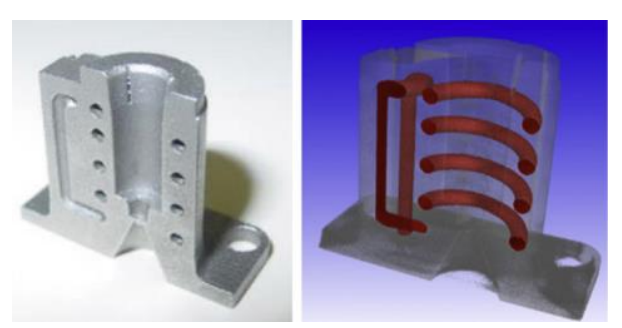

(a)

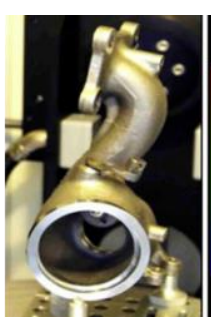

.

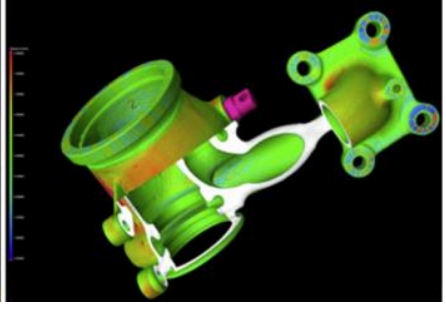

(b)

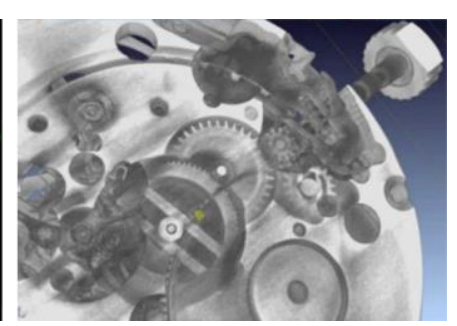

(c)

Figure 1: XCT examples: (a) illustrates a cross section of a nozzle, where the internal channels are made visible by XCT; (b) a cast iron object measured with XCT with CAD-compare of XCT voxel model and nominal CAD model; (c) analysis of an interior watch mechanism, showing all the components of the correspondent cross section $[4,5]$.

According to the National Institute of Health Consensus Development Conference, an implant is "a medical device made of one or more biomaterials intentionally placed within the body, either totally or partially buried beneath an epithelial surface" that can be in contact with tissue for a significant period of time [7]. Biomaterials are "any substance (other than a drug) or combination of substances, synthetic or natural in origin, which can be used for any period of time, as a whole or as a part of a system which treats, augments or replaces any tissue, organ, or function of the body". These are materials that, according to the National Institute of Health Consensus Development Conference, are characterized for interfacing with biological entities [6,8].

Regarding biomaterial four classes can be mentioned: acellular tissue matrices, metallic materials, ceramic materials and polymers. Here we focus on metallic biomaterials. Metallic biomaterials are classified as inert materials because they provoke minimal tissue response, providing mechanical reliability, strength, stiffness, toughness and impact resistance [6]. In this paper, a dental cast model with 8 implants all connected by a removable screw bar is the object of study. Figure 2 depicts the referred component. The model is made of titanium, the most commonly used metal in medical applications. Titanium has one of the highest strength-to-weight ratios and corrosion resistances among metals. Moreover, it has excellent biocompatibility, thus high chance of acceptance by the human body.

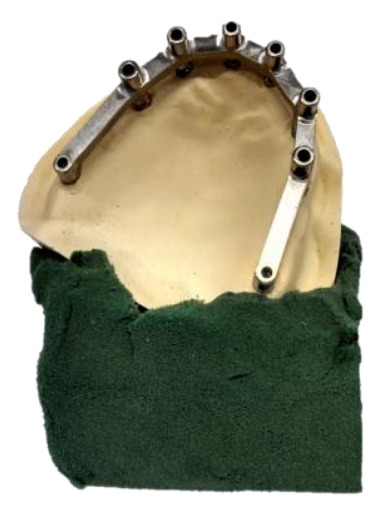

(a)

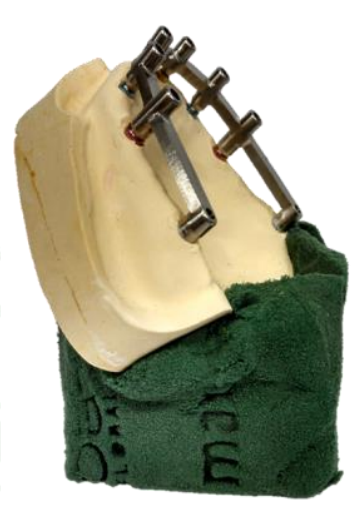

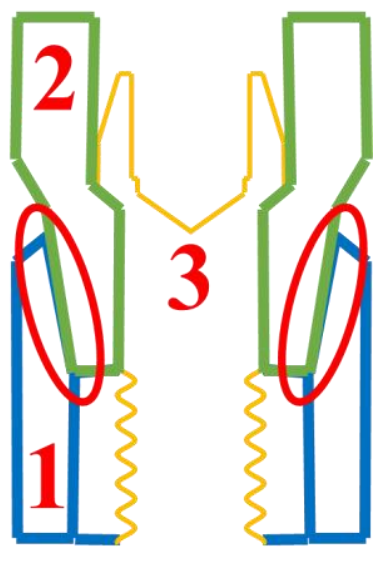

(b)

Figure 2: (a) Gypsum cast model and (b) the implant attached the bone structure (1), the retained bar (2) and the screw connecting both components (3).

XCT is since long being used in the medical field, be it to create detailed cross-section images of the human body that can aid the diagnostic of infections, identify masses, tumors, etc., or to aid in the design of customized medical implants such as mandible implants [6], chin implants [9], for the reconstruction of damaged jawbone [10], for cranial implants [11], etc. These customized free form implants impose a challenge when it comes to quality control since not all features are accessible by traditional contact or contactless measurement techniques, such as tactile coordinate measuring machines (tactile CMM) and laser line scanners (LLS), especially when it comes to hidden features. In this sense, XCT 
offers many opportunities. It is the only measurement technique capable of measuring both inner and outer features of an object in a non-destructive way. Assessing the quality of custom-made implants in terms of their mechanical and geometrical properties is of extreme importance. The preoperative planning or surgical simulation is prone to fatal errors in case the medical model is not sufficiently accurate [12]. Moreover, medical devices such as implants are associated with a definitive risk of bacterial and fungal infections $[13,14,15]$.

In order to reduce the risk of infections in dental implants that may start at the mating interface between the implants and the retained bar, a verification of the assembled structure is important for the validation of the designed bar structure. Measuring the components of an assembly separately does not guarantee proper functioning of the assembly. Geometry and dimensions of the components may differ in unassembled and assemble state. For example, an assembly may fail geometrically even if all individual elements of the assembly meet the tolerances when unassembled. Moreover, mismatches and undesired gaps or collisions between individual components of the assembly may possibly happen due to unwanted deformations of those components enforced by the assembly $[3,4]$.

In this paper, we investigate the mating interface of the dental implant illustrated in Figure 2 through XCT. The fitting between both the implant and the retained bar should be good enough to avoid gaps after the retained bar is screwed onto the implants. Deformation of the conical mating structure may happen during the assembly due to pressure exerted by the screw. Moreover, flaws in the design could also lead to gaps in that region. The diagram in Figure 2 (b) exemplifies the aforementioned. In Figure 2 (b) the part in blue (1) represents the implant whereas the retained bar is depicted in green (2). The yellow component (3) is the screw that connects the other two components. The study here is thereby focused on the connection between the blue and green part illustrated by the red circles where gaps might possibly appear.

\subsection{Principles of X-ray computed tomography}

In XCT, a source generates X-rays that will travel to the X-ray sensor (detector). An object, positioned between the source and the detector, will attenuate the X-rays travelling their way to the detector. This attenuation is due to the material absorbing and scattering X-rays, which depends on three factors: the length of the penetration through the object, the composition and density of the object and the energy of the X-rays leaving the X-ray tube. The detector measures the X-ray attenuation and a two-dimensional image (radiograph) of the object is created. Figure 3 depicts the principles of XCT.

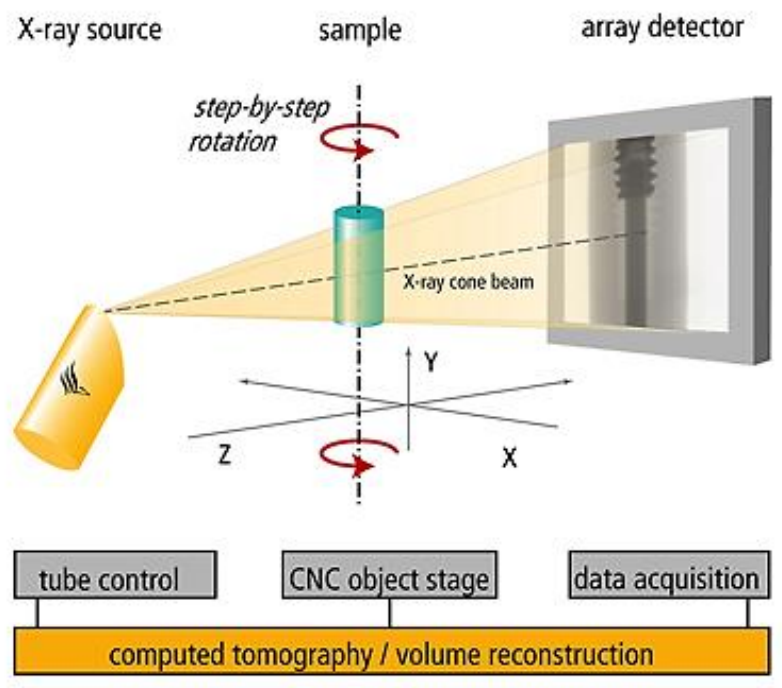

Figure 3: Principle of X-ray computed tomography. The X-ray tube generates X-rays that travel to the detector and are attenuated by any object placed in between the two components. The detector senses the attenuation creating a gray level image (commonly known as radiograph) [16].

In order to use tomography on an object, several hundreds to a few thousands of such two-dimensional radiographic images are made. The sequence of image is made while the object rotates within the X-ray cone beam in numerous 
accurately known rotational positions. Using suitable mathematical methods (such as filtered back projection), a volume model that describes the entire geometry and material composition of the workpiece can be calculated from this sequence. Due to the beam shape, this process is called cone beam tomography $[3,17]$.

The volumetric model represents the scanned object in a 3D environment filled with voxels. A voxel is a 3D analogue of a pixel. The voxel is used in tomography to describe the local radiation absorption in an equidistant spatial array. Dark voxels represent areas with low absorption whereas bright voxels are areas with high absorption [17].

The voxel size represents the theoretical physical resolution of a scanned object and it will influence the final resolution of a CT system. Resolution can be defined as the smallest detail that is still perceptible by a measuring device. In XCT the resolution can be adjusted by moving the object either closer to the source (higher image magnification and pixel resolution, but more blurring) or closer to the detector (sharper images, but lower resolution). Diving the detector pixel size by the magnification factor $M$ of the scanned object yields the theoretical physical resolution $R_{V S}$. [3,17,19]

$$
R_{v s}=\frac{\text { pixelsize }_{\mathrm{det}}}{M}
$$

Dimensional quality control requires high spatial resolution and accuracy in respect of the rules of measurement uncertainty and traceability to the SI unit of measurement, the meter. Many factors influence the spatial resolution of CT reconstructions, including: focal spot size of the source, performance of the detector, magnification, number of projections, reconstruction algorithms, and data post-processing. The focal spot size is particularly important in determining the image quality. The smaller the spot size, the sharper the edges of an object will be. In case of large spot sizes, blurring will occur. [3,4]

The 3D reconstruction of a scanned object is followed by the edge (surface) detection or segmentation determining the respective interfaces between solid materials and surrounding air or between two different solid materials. The edge detection converts 3D voxel data into 3D surface data. The threshold method greatly influences the resolution (sub-pixel resolution). Edge detection is often based on gray value thresholding: a certain gray value is assumed to coincide with the transition from one material to another. However, various other techniques exist to identify object or material edges: assigning a threshold gray value to "edge voxels", interpolation between voxel gray values, search for maximum gray value derivatives, mid gray value between the light air voxel and the dark material voxel levels (Figure 4 b, bottom right), local adaptive gray threshold, etc. [3,17,19]

The edge gray value is very dependent on the material being irradiated and obviously on the intensity of the radiation. Inter-voxel interpolation is needed to locate the edge with sufficient accuracy within edge transition voxels. This interpolation increases the resolution to sub-voxel resolution, but does not necessarily improve accuracy, unless a proper gray value calibration is performed [4].

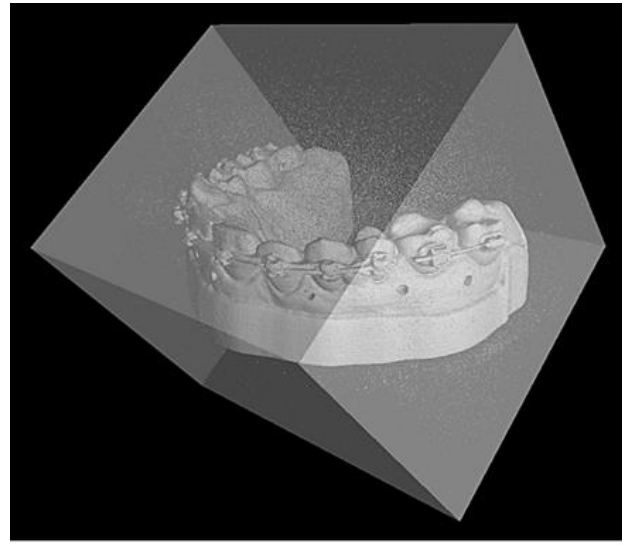

(a)
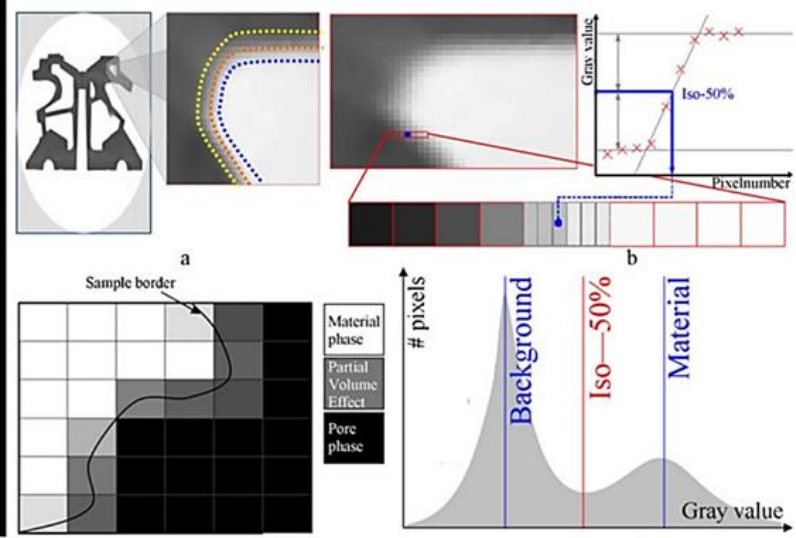

(b)

Figure 4: (a) illustrates the voxel cloud after the reconstruction of a scanned object. The object itself is embedded in voxels that represent its surrounding air during the acquisition phase. (b) depicts the segmentation stage where material and air are segmented converting 3D voxel data into 3D surface data [3]. 


\section{THE WORKPIECE: GYPSUM CAST MODEL}

\subsection{Comparison among different measurement techniques}

The dental gypsum cast model contains 8 implants which are connected by a removable screwed retained bar. The retained bar includes a mating interface connection that should ensure a perfect fitting when the connection is fixed with screws. The analysis consists of the measurement of 6 inner and 6 outer diameters of the dental implant and the mating interface between the implants and the retained bar (Figure 2 b). A comparison among tactile CMM, optical scanner based on fringe projection (GOM Atos Triple $8 \mathrm{M}$ ) and XCT for the diameters of the implants is also performed. Figure 5 illustrates the measurands and the workflow for XCT.
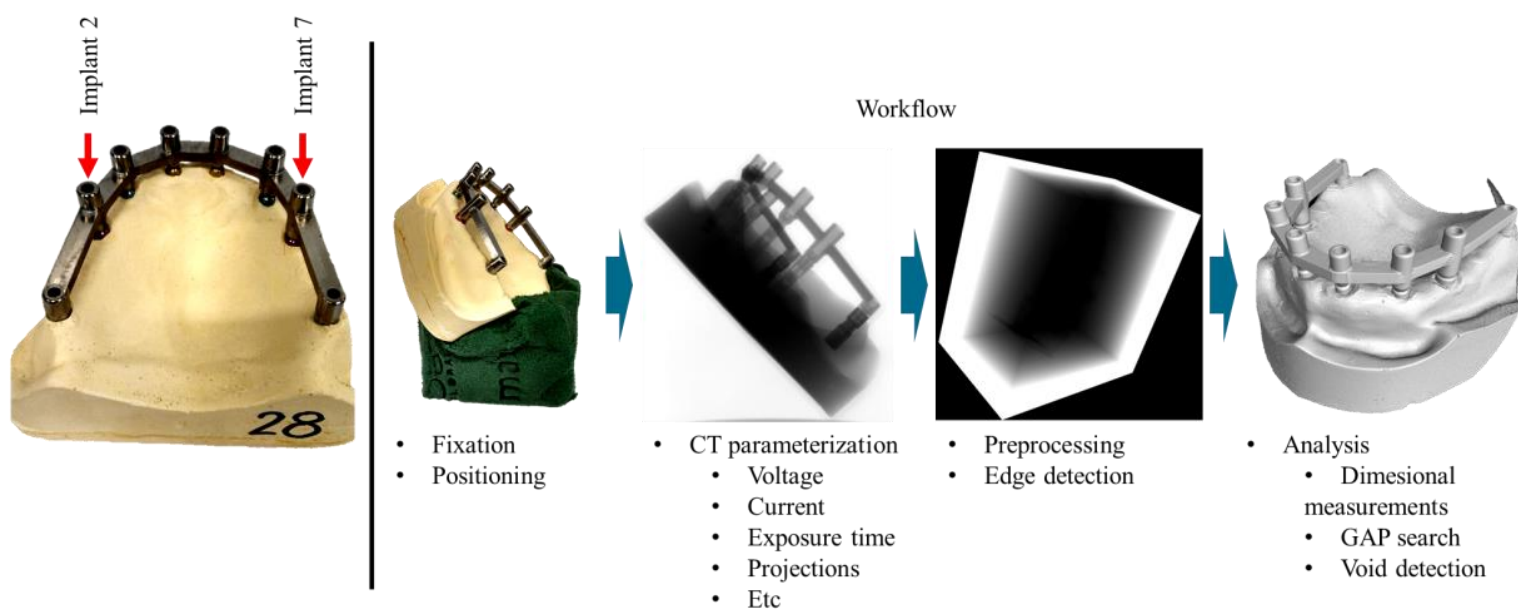

Figure 5: The gypsum cast model with its implants and the workflow for the XCT data analysis. All implants from 2 to 7 were measured with 3 different measurement techniques (tactile CMM, optical scanner and XCT) and their results compared to each other.

The tactile CMM measurements were done with a Mitutoyo FN905 system. Each measurement was repeated 3 times, each repetition using a total of 21 points. As every implant of the dental cast model has a different orientation, clay was used to provide a rough alignment between the cylinders and the granite table of the machine. This technique was used for every single implant. In this way, it was possible to avoid that the shaft of the tactile probe would touch the cylinder being measured rather than the probe itself, which would compromise the measurement results. Figure 6 illustrates the system used to acquire the reference values of the inner and outer diameter as well as the clay solution applied to compensate differening implant orientation.

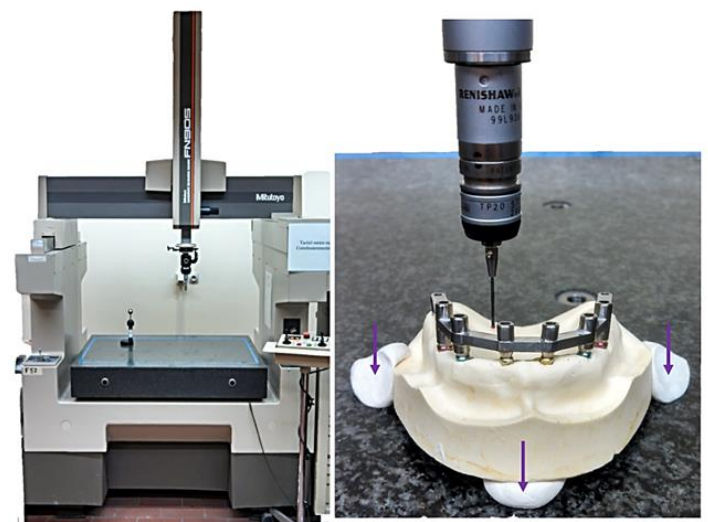

(a)

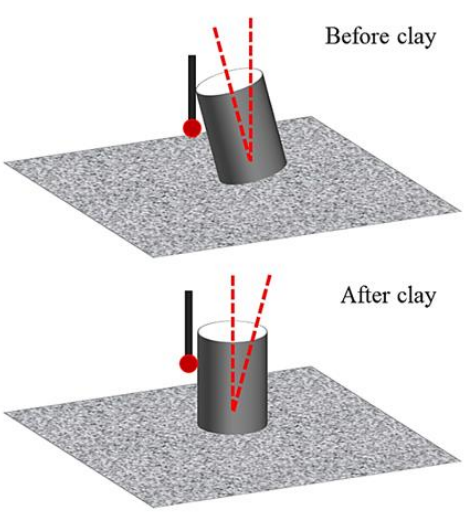

(b)

Figure 6: (a) the tactile CMM Mitutoyo FN905 system used to acquire reference values for the inner and outer dimeter of the implants. (b) adopted solution to compensate the orientation of the implants. 
The most important disadvantages of optical scanners based on triangulation, such as fringe projection, when compared to tactile CMMs, is the limited accuracy that is strongly influenced by surface quality. It is difficult and sometimes even impossible to measure shiny surfaces, e.g. machined steel or aluminum, because specular reflection is needed in order to capture the projected light with the camera. The latest scanners are better equipped to deal with this problem but the accuracy for shiny surfaces will always be lower than for diffusely reflecting surfaces. Therefore, metal parts are often sprayed with a diffuse reflecting coating before they are measured [20]. This thin layer of spray adds an additional uncertainty to the measurement which is difficult to be compensated for. The thickness of the spray is hard to be estimated and not entirely equal for the entire part since it is often dependent on the tester who applied the coating.

Regarding XCT, beam hardening may disturb correct edge detection leading to an edge offset, as it alters the gray value of edge pixels. Beam hardening refers to the fact that low energy photons are more rapidly attenuated than high energy photons. This gives the false impression that the outer part of a scanned object is more absorptive than its core as the capturing detectors counts incident photons independent of their energy. This non-linear attenuation of the X-rays happens due to the polychromatic character of the spectrum and the energy dependent X-ray attenuation. [3,21]

\subsection{Dimensional measurement results}

Figures 8 and 9 depict the results for the comparison for the three techniques used to measure the outer and inner diameter of implants 2 to 7 (as illustrated in Figure 7). The big deviation between the tactile CMM results and the optical scanner can relate to both the diffuse spray coating as well as to the physical difference between tactile probing (which filters shallow valleys of the surface roughness) and optical probing (which allows light to enter even these shallow valleys) [22]. As can be seen from the graphs, there is a minimum and maximum deviation of respectively 23 and $27 \mu \mathrm{m}$ between the tactile CMM and the optical scanner for the outer diameter, whereas for the inner diameter the minimum and maximum deviation vary from $9 \mu \mathrm{m}$ to $24 \mu \mathrm{m}$. As for the measurement with the XCT system, these deviations vary from 2 to $10 \mu \mathrm{m}$ for the outer diameter, and from 6 to $16 \mu \mathrm{m}$ for the inner diameter.

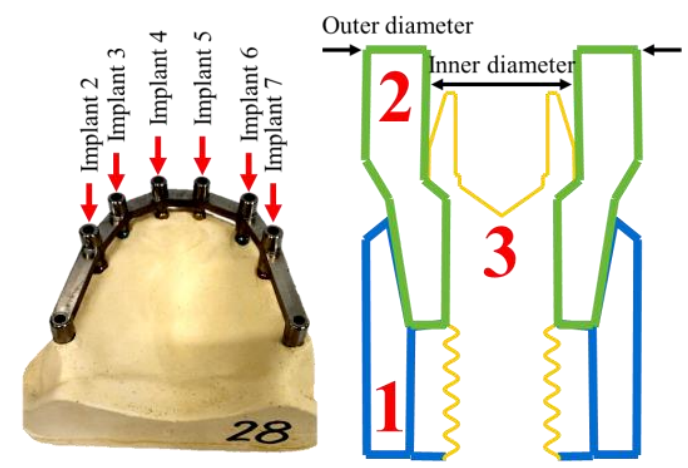

(a)

(b)

Figure 7: (a) implants from 2 through 7 of the cast model; (b) measurands: inner and outer diameter of implants 2 through 7.
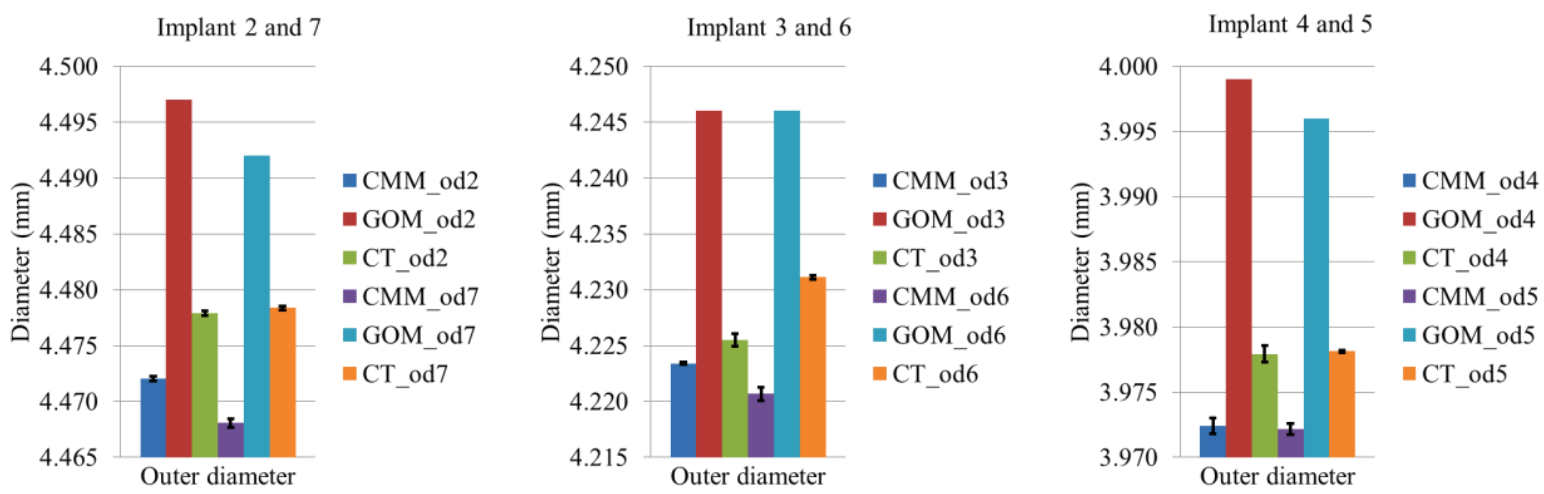

Figure 8: Comparison for the outer diameter (od in the graph) of all 6 implants: tactile CMM, optical scanner (GOM) and $\mathrm{XCT}$. 

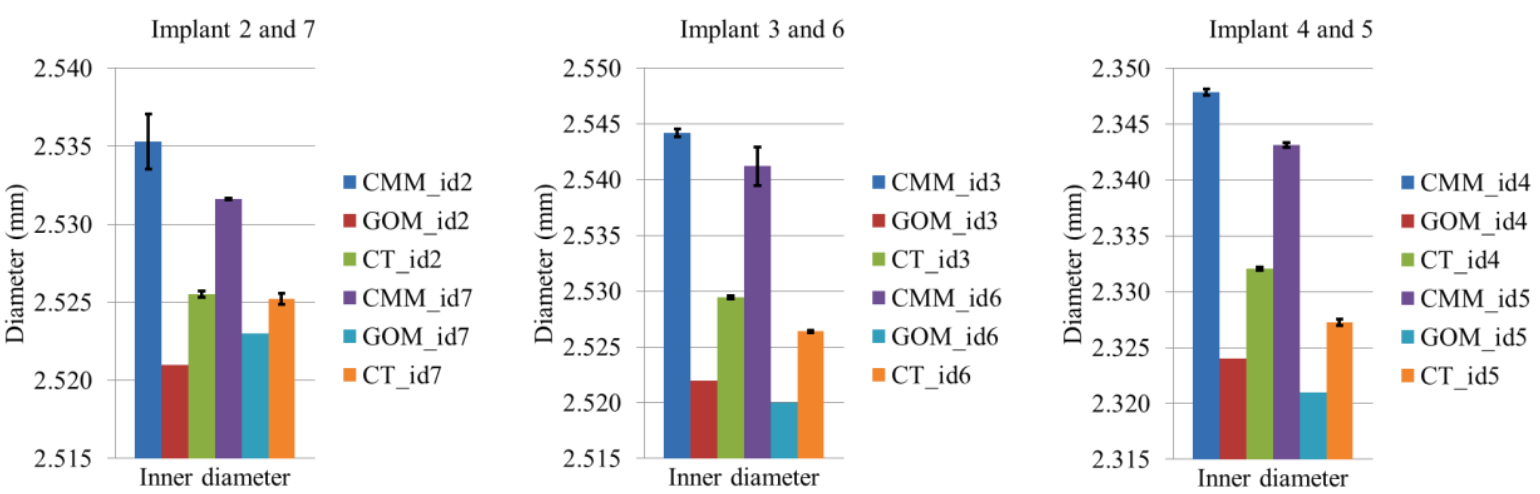

Figure 9: Comparison for the inner diameter (id in the graph) of all 6 implants: tactile CMM, optical scanner (GOM) and XCT.

\subsection{Edge offset compensation}

The accuracy of XCT is greatly influenced by three main factors; the accuracy of the 2D radiographs, the accuracy of reconstructing the $2 \mathrm{D}$ radiographs into a $3 \mathrm{D}$ model and errors when performing the measurements on the $3 \mathrm{D}$ model. The first two are highly dependent on geometrical offsets and misalignments of the XCT system. Geometrical offsets and misalignments of the CT system will produce edge offsets of the CT data when compared to tactile CMMs. Such offsets are usually treated as systematic errors and can be compensated for. However, one should notice that inner and outer edge offsets have to be calculated independently from each other, since a main contributor - beam hardening - affects both inner and outer structures differently. A common way of identifying edge offset errors usually relies on the measurement of a second workpiece, commonly of the same material as the object of interest, containing calibrated reference values. By evaluating what happens to the reference object when submitted to the same measurement conditions as the object of interest, edge offset errors can be extracted and applied to the object of interest. Figures 10 and 11 show both inner and outer diameters of the measured implants before and after offset compensation.
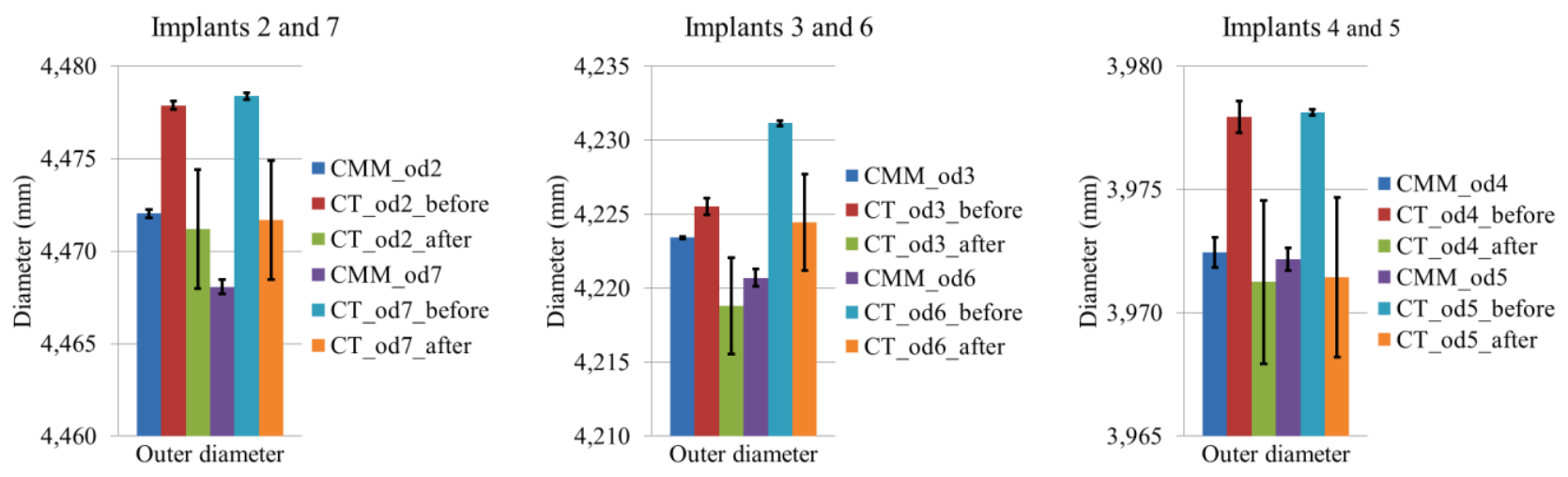

Figure 10: Compensation of systematic errors (offset errors) for the outer diameters of implants 2 through 7. The higher deviation bar comes from the combination of the measurement uncertainty of both tactile CMM (the reference system) and the XCT measurements. 

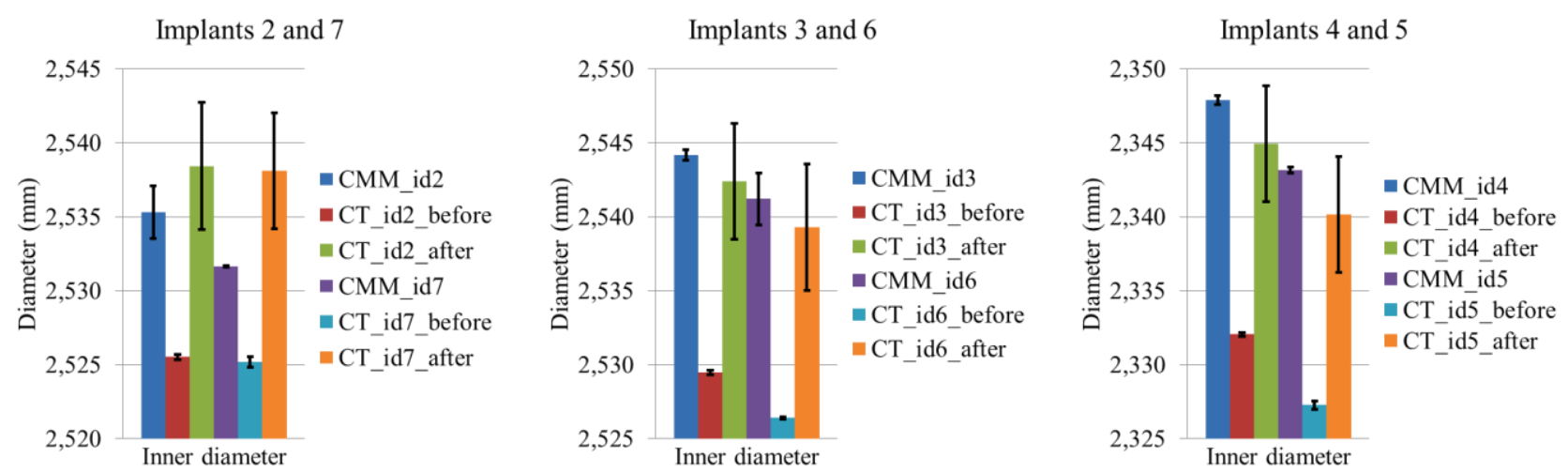

Figure 11: Compensation of systematic errors (offset errors) for the inner diameters of implants 2 through 7. The higher deviation bar comes from the combination of the measurement uncertainty of both tactile CMM (the reference system) and the XCT measurements.

\subsection{Investigating the connection between the implants and the retained bar}

Initially all XCT scans of the cast model were done at a magnification of 5 times, leading to a resolution in terms of voxel size of $40 \mu \mathrm{m}$. At this point due to the limited resolution (voxel size) and partial volume effects (spatial resolution), gaps at the connections were not found. However, as can be seen in Figure 12, looking at the cross section image of the scanned implants, it was possible to identify a dark gray threshold line which was representing the interface between the two components. Moreover, the algorithm for segmentation was able to identify a small portion of edges for implants 2, 3 and 5 which supports the idea that the gray line seen in Figure 12 could mean gaps at the connection.

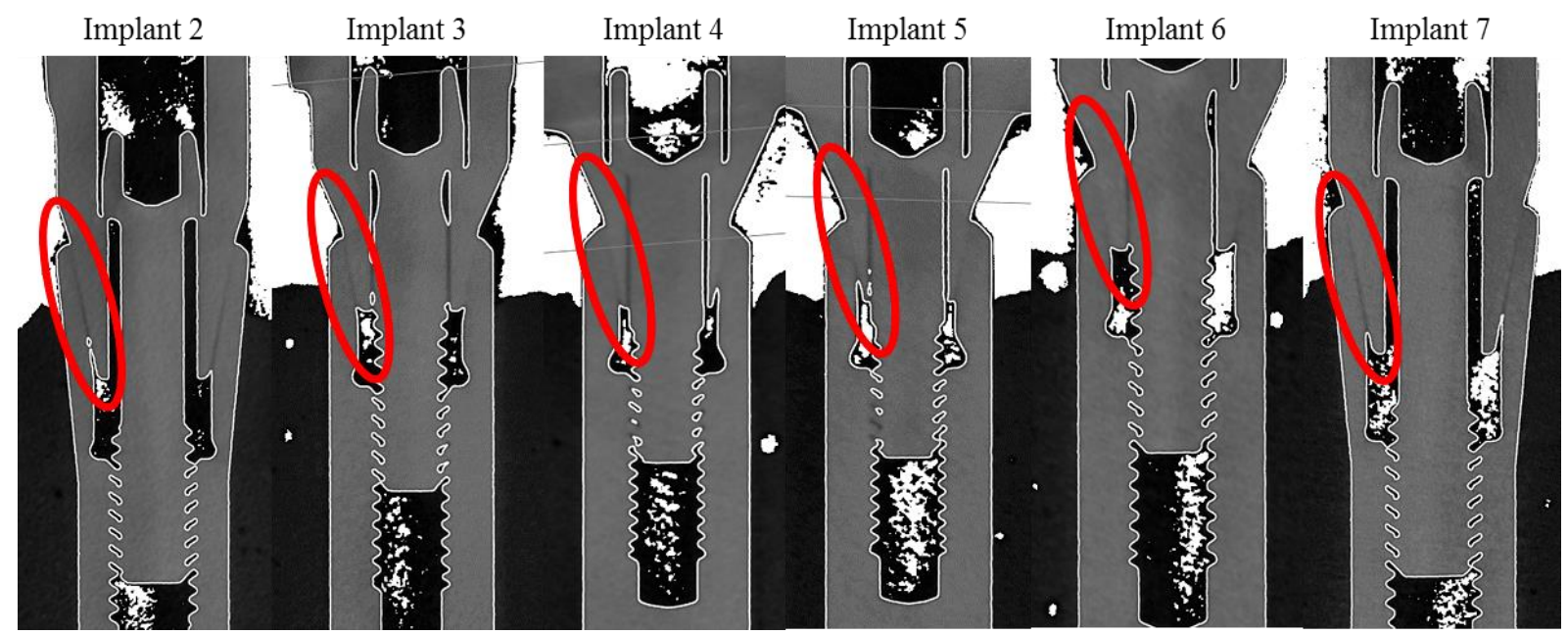

Figure 12: Looking for gaps from implant 2 to 7. First results at a magnification of 5 times were promising once a gray level transition between the components was observed as illustrated by the red markings in the image.

With the evidence of gaps between the connections of implants 2, 3 and 5, a ROI measurement for implants 2, 3 and 4 was conducted. With this ROI measurement, it was possible to increase magnification and, therefore, the measurement resolution. The magnification was raised from 5 to 10 and the resulting voxel size lowered from $40 \mu \mathrm{m}$ to $20 \mu \mathrm{m}$. Higher magnifications were not possible due to the size of the workpiece and positioning within the X-ray cone beam.

As can be seen in Figure 13, an expressive gap was found at the connection of implant 2. 


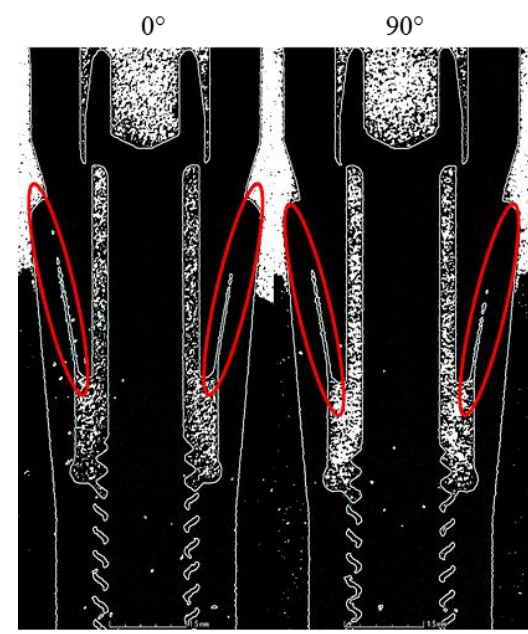

a) implant 2

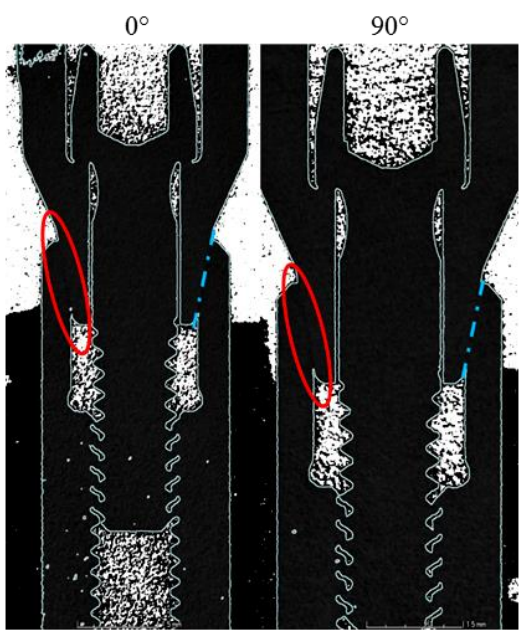

b) implant 3

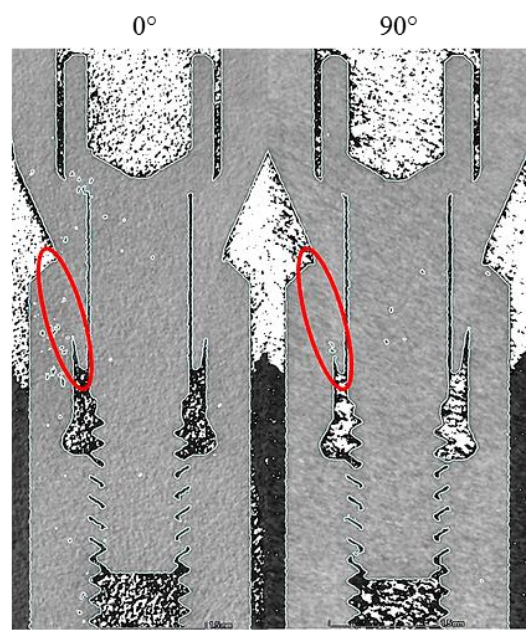

c) implant 4

Figure 13: a) gaps found at implant 2 due to a higher measurement resolution. b) the resolution of $20 \mu \mathrm{m}$ was still not enough to detect or exclude possible gaps at implant 3.

In order to get an estimation of the gap, two circles were fitted at the bottom of the connection. Then, by subtracting both diameters, and diving the result by 2 , it was possible to obtain an estimation of $53 \mu \mathrm{m}$ for the gap present at the connection of implant 2 (Figure 13 a).

For implants 3 and 4, even with higher magnification it was not possible to detect any gaps at the connections, which might indicate the absence of gaps between the components or, a limited resolution which makes the search for gaps infeasible. However, when analyzing for instance Figure 12, we notice that every time there is contact between the two metallic components, it is shown in the image as the continuation of the component itself with no difference in the gray level during the transition. Whereas when there is a small space between them, it is shown as a darker gray level (tending to black, which represents air), indicating that there might exist space between those components. The fact that they are not represented in the image with their respective edges, only means that we could not achieve enough resolution so that the segmentation algorithm could identify the edges of both components. Figure 14 depicts the aforementioned explanation.

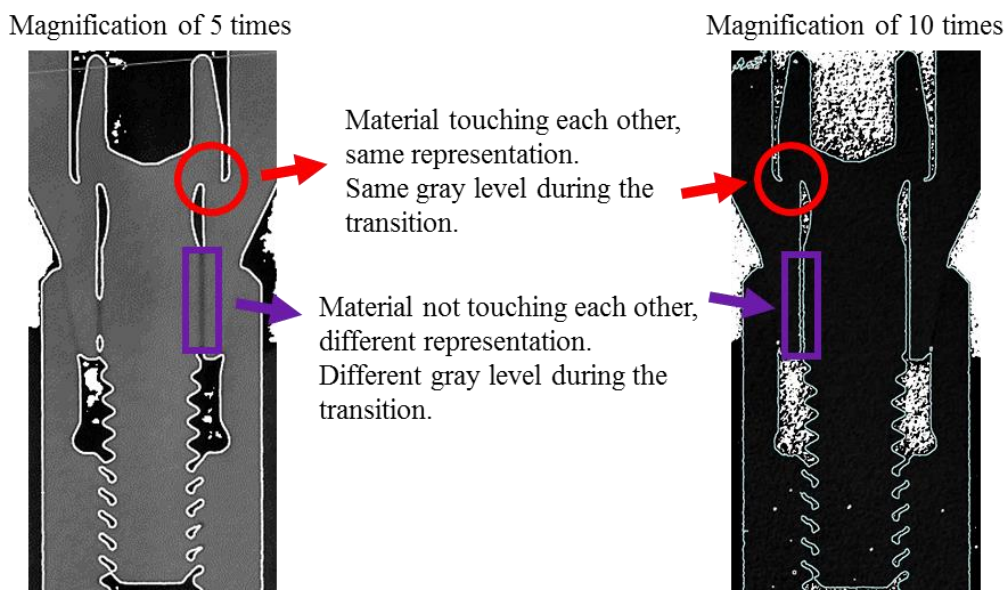

Figure 14: Gray level representation between the transition of the two metallic components, where higher magnification (higher resolution) allowed the segmentation algorithm to identify the edges between the two components.

That been said, when analyzing again Figure 12, it is possible to observe that the aforementioned phenomenon occurs for all 6 implants. Hence meaning that there could indeed exist a small gap at the connections of the two components that could not be seen due to the lack of measurement resolution. 
To support the idea that gaps can be identified by the gray level transition between two components, a histogram analysis was performed where supposedly a gap is present. The analysis represents a linear section of which the gray level values are plotted along this line. Figure 15 depicts this analysis which was performed at different heights of the connection, moving from the bottom to the top (blue dashed lines). As can be noticed, air is identified at the connection and starts to vanish as the height, for the following profile, is increased, up to a point that only material is identifiable (fifth profile line). This happens because of the limited resolution. As the height is increased, the gap becomes smaller and less voxels are used to represent it. In the fifth histogram, for instance, even if still there is a gap between the two components, it will not be identifiable since the resolution is at this stage limited to $20 \mu \mathrm{m}$.

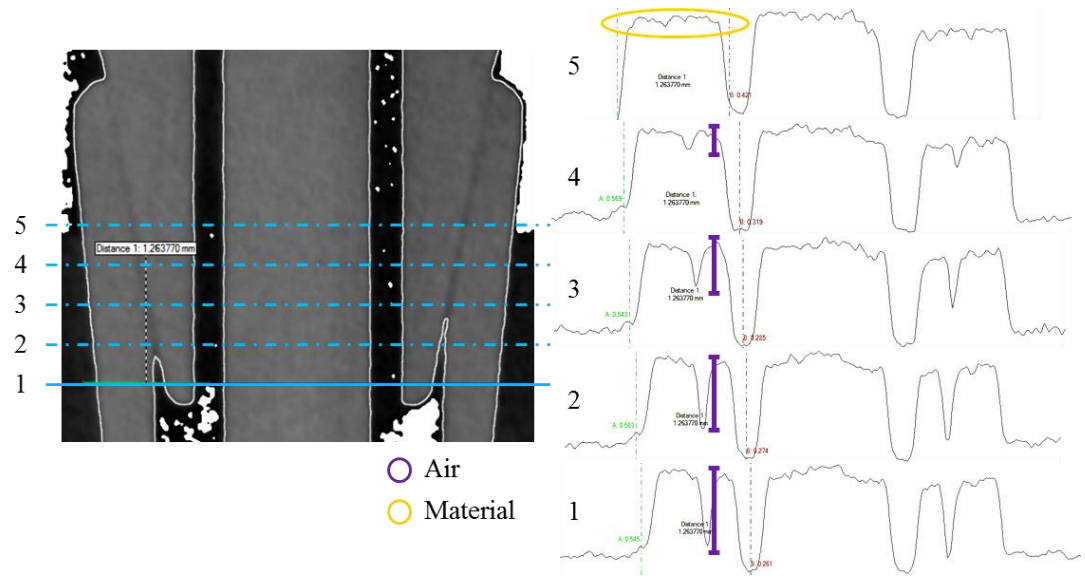

Figure 15: Gray level profile analysis of the transition between the two metallic components at different heights. Although the segmentation algorithm, due to limited resolution, did not detect edges, it is possible to confirm, due to the line profile, that gaps are present at the connections.

\section{CONCLUSIONS}

The number of industrial applications of XCT is high and rapidly increasing. However, the technology still possesses limitations and problems in solving some of the industrial challenges as illustrated in this paper. The accuracy of XCT is greatly influenced by three main factors; the accuracy of the $2 \mathrm{D}$ radiographs, the accuracy of reconstructing the $2 \mathrm{D}$ radiographs into a 3D model and errors when performing the measurements on the 3D model.

As demonstrated, limited resolution due to either voxel size or spatial resolution can compromise the analysis of a data set. The size of the workpiece limits spatial resolution hence hindering identification of gaps that are smaller than one voxel. Increasing spatial resolution in XCT is often difficult since it is intrinsically related to the object to be measured. Depending on size, geometry and material, the CT practitioner has to choose an X-ray energy that provides full penetration of the object at all orientations. However, higher energies will increase the focal spot, where the X-rays are generated, hence decreasing spatial resolution.

This paper, also illustrates that identification of flaws smaller than the voxel size is achievable by histogram analysis. The gray level transition, which happens where the two components supposedly touch each other, represents a qualitative parameter to identify the presence of gaps at the connections.

Hence, XCT metrology is proven to yield some major benefits over other CMM inspection methods. XCT is today the only technology that allows assessing internal structures in a non-destructive way. This is of particular importance for complex cast objects or additive manufactured components that often involve inner geometries that are inaccessible for conventional techniques. XCT moreover allows to measure components in the assembled state. This is important as dimensions may differ between the non-assembled and the assembled state. Moreover, it is the only technology that allows all of the aforementioned inspection methods within only one quality inspection job. [3,4] 


\section{REFERENCES}

[1] Bartscher M, Hilpert U, Härtig F, Neuschaefer-Rube U, Goebbels J, Staude A, "Industrial Computed Tomography, an Emerging Coordinate Measurement Technology with High Potential," Proc. of NCSL International Workshop and Symposium, (2008).

[2] Mitchell K W, [A Generalized Approach to Wall Thickness Measurements in CT Images] Topical Proc. Industrial Computerized Tomography, ASNT, 120-124. ISBN 0-931403-89-8, (1989).

[3] Kruth J-P, Bartscher M, Carmignato S, Schmitt R, De Chiffre L, Weckenmann A, "Computed Tomography for Dimensional Metrology," CIRP Annals 60(2), 821-842 (2011).

[4] De Chiffre L, Carmignato S, Kruth J-P, Schmitt R, Weckenmann A, "Industrial Applications of Computed Tomography," CIRP Annals 63, 655-677 (2014).

[5] Nikon, "Mobile phones, shavers \& watches," http://www.nikonmetrology.com/en_EU/Applications/ConsumerGoods-White-Goods-Household-Applications/Mobile-phones-shavers-watches (15 March 2016).

[6] Bartolo P, Kruth J-P, Silva J, Levy G, Malshe A, Rajurkar K, Mitsuishi M, Ciurana J, Leu M, "Biomedical production of implants by additive electro-chemical and physical processes," CIRP Annals 61, 635-655 (2012).

[7] Bandyopadhyay A, Krishna BV, Xue W, Bose S, "Application of Laser Engineered Net Shaping (LENS) to Manufacture Porous and Functionally Graded Structures for Load Bearing Implants,” Journal of Materials Science Materials in Medicine 20, 29-34 (2009).

[8] Williams D F, [Definitions in Biomaterials. Proceedings of a Consensus Conference of the European Society for Biomaterials], Elsevier 4, (1987)

[9] Singare L, Dichen L, Bingheng G, Zhenyu L, Yaxiong, "Customized design and manufacturing of chin implant based on rapid prototyping," Rapid Prototyping Journal 11(2), 113-118 (2005).

[10] Radovan H, Jozef Ž, Richard L M, “Applications of Metrotomography in Biomedical Engineering, Biomedical Engineering - Technical Applications in Medicine”, ISBN: 978-953-51-0733-0, InTech, DOI: 10.5772/50713 (2012).

[11] Hudak R, Lisý M, Majernik J, Tóth T, Zivcák J, "Usage of Industrial Computed Tomography for Evaluation of Custom-Made Implants," ISBN: 978-3-319-19146-1, DOI: 10.1007/978-3-319-19147-8_2 (2015).

[12] Salmi M, "Medical applications of additive manufacturing in surgery and dental care," Doctoral dissertation Aalto University, (2013).

[13] von Eiff C, Jansen B, Kohnen W, Becker K, "Infections associated with medical devices,” Drugs 65(2), 179-214 (2005).

[14] Cyteval C, Bourdon A, "Imagerie des infections sur matériel orthopédique," Journal de Radiologie Diagnostique et Interventionnelle 93(6), 578-589 (2012).

[15] Pyea A D, Lockhartb D E A, Dawsona M P, Murrayb C A, Smithb A J, “A review of dental implants and infection," Journal of Hospital Infection 72(2), 104-110(2009).

[16]GE, "Measurements and control solutions," http://www.phoenixxray.com/en/company/technology/principles_of_operation/principle_060.html, (16 March 2016).

[17] Christoph, Ralf a Hans Joachim NEUMANN. [X-ray Tomography in Industrial Metrology]. ISBN 978-3-86236045-1, (2012).

[18] Welkenhuyzen F, Kiekens K, Pierlet M, Dewulf W, Bleys P, Kruth J-P, Voet A, "Industrial computer tomography for dimensional metrology: Overview of influence factors and improvement strategies. Proceedings of the 4th International Conference on Optical Measurement Techniques for Structures and Systems," Optimess2009 International Conference on Optical Measurement Techniques for Structures and Systems, 401-410 (2009).

[19] Cantatore A, Müller P, "Introduction to computed tomography," Department of Mechanical Engineering - DTU: Report, (2011).

[20] Van Gestel N, Cuypers S, Bleys P, Kruth J-P, "A performance evaluation test for laser line scanners on CMMs," Optics and lasers in engineering 47, 336-342 (2009).

[21] Dewulf W, Tan Y, Kiekens K, "Sense and non-sense of beam hardening correction in metrology," CIRP Annals 61, 495-498 (2012).

[22] Boeckmans B, Tan Y, Welkenhuyzen F, Guo Y, Dewulf W, Kruth J-P, "Roughness offset differences between contact and non-contact measurements," International conference of the european society for precision engineering and nanotechnology, 189-190 (2015). 